\title{
Misurazione costi-performance: casi aziendali a confronto
}

\author{
Luciano Marchi
}

Il sistema di misurazione delle performance è influenzato dal rapporto tra scelte di gestione e sistema di controllo, nei termini indicati nel precedente editoriale della rivista curato da Rosa Alba Miraglia (Management Control, n. 2, 2012), perché il sistema di misurazione delle performance è un sub-sistema dello stesso sistema di controllo. Pertanto, l'evoluzione subita nel tempo dal sistema di misurazione deriva dai cambiamenti che intervengono nella "filosofia" aziendale e che influenzano anche il sistema di controllo.

Il suddetto legame del sistema di controllo con la gestione aziendale si realizza, ovviamente, anche quando il sistema delle misurazioni viene applicato non alle performance ma ai costi delle risorse utilizzate e delle produzioni realizzate in ambito aziendale.

Così come avviene per il sistema di controllo, anche il sistema di misurazione dei costi e delle performance si adegua alle necessità strategiche tese a perseguire il vantaggio competitivo. Ne consegue anche, come rileva la Miraglia, "che il sistema di misurazione dovrebbe avere una struttura multidimensionale, cioè accogliere misure quantitative e qualitative; misure finanziarie e non finanziarie”.

Come il precedente numero della rivista, anche questo numero è centrato sulla progettazione del sistema di misurazione dei costi e delle performance. Tutti i contributi presentati aderiscono inoltre alla tradizionale tesi del rapporto causale tra gestione e sistema di controllo.

Nel primo contributo di ricerca, realizzato da Marco Giuliani, si analizza il rapporto tra la variabile temporale e la sua misurazione da due diverse prospettive: 1) la capacità dei metodi e degli strumenti di management accounting di "catturare" la dimensione temporale; 2) la "costruzione" di un concetto e di una dimensione temporale economico-aziendale.

Il punto di partenza della ricerca è l'analisi dello stato dell'arte, dove il tempo adottato normalmente è quello di tempo fisico e sono spesso trascurati i pro- 
fili qualitativi del tempo e di come i processi di accounting possono contribuire a costruire specifici aspetti della dimensione temporale.

Con il ricorso ad un caso aziendale con approccio interventista, l'autore evidenzia come l'utilizzo delle misure di tempo, inserite o meno in un sistema integrato di misurazione delle performance, contribuisca a sviluppare la percezione della sequenza (relazioni causa-effetto) e durata delle attività aziendali; favorisca inoltre interscambi tra soggetti o gruppi con percezioni diverse e permetta di sviluppare una visione comune della dimensione temporale. Si determina dunque un circolo virtuoso tra accounting e dimensione temporale.

I due successivi contributi di ricerca sono centrati sulla misurazione delle performance e sulla valutazione manageriale nell'ambito delle aziende sanitarie. Queste problematiche richiamano, infatti, una crescente attenzione da parte degli studiosi, del legislatore e degli operatori che, a vario titolo, sono coinvolti nel settore sanitario.

Il primo contributo, fornito da Adele Caldarelli, Marco Maffei e Rosanna Spanò, si focalizza sulle problematiche concernenti l'implementazione dei sistemi di valutazione della performance dei Direttori Generali delle aziende sanitarie italiane e intende dare risposta alla seguente domanda: $i$ sistemi di valutazione della performance dei Direttori Generali - intesi come archetipi in grado di assimilare le influenze dall'ambiente esterno e di trasferirle allo schema interpretativo della Regione - hanno effettivamente prodotto un cambiamento sostanziale nei Servizi Sanitari Regionali Italiani per la creazione e la condivisione di una cultura del controllo?

Per rispondere a tale interrogativo la ricerca di Caldarelli, Maffei e Spanò si avvale di una meaning oriented content analysis dei 21 Piani Sanitari predisposti dalle Regioni italiane, "vista la primaria importanza che questi documenti triennali di pianificazione assumono per la comunicazione di obiettivi, modalità per raggiungerli e di parametri e indicatori in base a cui svolgere la valutazione”.

Il successivo contributo di ricerca, fornito da Antonio Leotta e Daniela Ruggeri, fa specifico riferimento alle aziende ospedaliere pubbliche nelle quali, da diversi anni, "si evidenziano dei fabbisogni di controllo dovuti a diffuse carenze di produttività e alla limitata efficacia nell'espletamento delle proprie funzioni”.

Le aziende ospedaliere pubbliche, come affermano ancora gli autori, "rappresentano un contesto multi-stakeholder, caratterizzato dalla compresenza di una logica medico-professionale e di una manageriale. La prima contempla attività di ricerca, di didattica universitaria e di assistenza sanitaria, che si riflettono su elevati costi di struttura, richiedendo interventi amministrativo-manageriali. In un tale contesto, i cambiamenti nei sistemi in esame si 
presentano largamente condizionati dalla dialettica che si instaura tra i diversi soggetti istituzionali rappresentanti le due logiche”, quella manageriale e quella medico-professionale.

Il framework teorico sviluppato nella parte iniziale del lavoro viene applicato ad un caso studio condotto presso un policlinico universitario siciliano. L'osservazione longitudinale copre il periodo compreso tra il 1998 e il 2004, durante il quale si avvicendano quattro diverse procedure di misurazione delle performance e di valutazione manageriale.

Nel quarto contributo di ricerca inserito nel presente numero della rivista, l'attenzione degli autori, Loris Landriani, Alessandro Scaletti e Luigi Lepore, si sposta dalla misurazione delle performance alla determinazione dei costi standard. Il quadro gestionale di riferimento è quello delle aziende dei trasporti pubblici locali.

Il quadro giuridico di riferimento è rappresentato invece dalla Legge 42/09 che ha introdotto in Italia il cosiddetto "federalismo fiscale" con l'obiettivo, come affermano gli autori, "di avvicinare i centri di spesa delle risorse pubbliche ai soggetti e ai territori che producono tali risorse, in modo da favorire non solo il controllo delle risorse stesse, ma anche il loro impiego efficiente ed efficace, in una parola, di migliorare l'accountability degli Enti Locali e delle relative aziende" prevedendo inoltre "la revisione delle regole di finanziamento per l'erogazione dei servizi, non più basate sulla spesa storica, ma sul calcolo di appositi costi/fabbisogni standard”.

Il lavoro di Landriani, Scaletti e Lepore si propone di indagare le modalità di calcolo dei costi standard per le aziende dei trasporti pubblici locali e le connesse problematiche di finanziamento e, quindi, di equilibrio economico, anche e soprattutto con riferimento ai vincoli sociali e non solo economici in senso stretto che caratterizzano a livello istituzionale le suddette realtà aziendali.

Tra gli spunti di riflessione che scaturiscono dal lavoro, un primo spunto riguarda il ruolo dei costi standard come strumento motivazionale. A tale riguardo, come affermano gli autori, "i costi standard dovrebbero rappresentare un obiettivo raggiungibile, altrimenti potrebbe addirittura essere un benchmark frustrante per le aziende, che potrebbero percepirlo come una norma imposta dall'alto e non come un meccanismo per la guida della gestione”.

Un secondo spunto di riflessione riguarda il significato del costo standard non come misura effettiva del costo, bensì come metodologia di allocazione delle risorse pubbliche, in quanto non devono generare disuguaglianze nelle diverse aree del Paese e non devono pregiudicare, con le parole degli autori, "le condizioni che dettano poi le effettive possibilità delle aziende operanti nel comparto di realizzare condizioni di equilibrio durevole ed evolutivo che si 
pongono alla base dell'autonomia aziendale nel settore pubblico come in quello privato".

Nell'ultimo lavoro, curato da Francesco Capalbo, l'attenzione si sposta dalla misurazione costi-performance alla rendicontazione con specifico riferimento all'accountability delle società partecipate dalle Pubbliche Amministrazioni italiane.

La conclusione a cui perviene l'autore è l'inadeguatezza del modello civilistico di rendicontazione in rapporto alle finalità di accountability assegnate alle società partecipate pubbliche. Il bilancio redatto secondo la normativa civilistica non presenta, infatti, il necessario livello di dettaglio, non permette il confronto dei risultati sugli obiettivi (pubblici) e, più in generale, non ha finalità immediatamente gestionali. 